\title{
Left Atrial Flutter After Segmental Ostial Radiofrequency Catheter Ablation for Pulmonary Vein Isolation
}

\author{
HAKAN ORAL, BRADLEY P. KNIGHT, and FRED MORADY \\ From the Division of Cardiology, Department of Internal Medicine, University of Michigan, Ann Arbor, Michigan
}

ORAL, H., ET AL.: Left Atrial Flutter After Segmental Ostial Radiofrequency Catheter Ablation for Pulmonary Vein Isolation. Segmental ostial ablation to electrically isolate pulmonary veins has been performed for atrial fibrillation. Left atrial flutter that utilized a critical isthmus adjacent to the ostium of the left superior pulmonary vein was diagnosed and successfully ablated in a patient 3 months after a successful pulmonary vein isolation procedure. Documenting the cause of symptoms after pulmonary vein isolation in patients with atrial fibrillation is critical in guiding therapy. (PACE 2003; 26:1417-1419)

atrial fibrillation, catheter ablation, pulmonary vein, atrial flutter

\section{Introduction}

Scar related left atrial flutter is a possible complication of arrhythmia surgery ${ }^{1-4}$ and linear ablation. ${ }^{5,6}$ However, to date there have been no reports of left atrial flutter occurring as a complication of segmental ostial ablation aimed at electrical isolation of a pulmonary vein. The purpose of this case report is to demonstrate that segmental ostial ablation is capable of creating the substrate for left atrial flutter.

\section{Case Report}

Segmental ostial ablation to isolate pulmonary veins was performed in a 55-year-old man who had a 10 year history of symptomatic, drug refractory, paroxysmal atrial fibrillation, occurring 15 times per month. Left ventricular ejection fraction was normal and left atrial diameter was $48 \mathrm{~mm}$.

After informed consent was obtained, a quadripolar electrode catheter (EP Technologies, San Jose, CA, USA) was inserted into the right femoral vein and positioned in the coronary sinus. Using a patent foramen ovale, a decapolar ring catheter (Lasso, Biosense Webster, Diamond Bar, CA, USA) and a deflectable, quadripolar electrode catheter (EP Technologies) with 2-5-2-mm interelectrode spacing and a 4-mm distal electrode with an embedded thermistor were introduced into the left atrium. Systemic anticoagulation was achieved with intravenous heparin to maintain an activated clotting time of 250-350 seconds.

Atrial fibrillation was induced by rapid atrial pacing in the coronary sinus. During atrial fibrillation, intermittent bursts of pulmonary vein tachy-

Supported in part by the Ellen and Robert Thompson Atrial Fibrillation Research Fund

Address for reprints: Hakan Oral, M.D., Cardiology, B1F245, 1500 E. Medical Center Dr., Ann Arbor, MI 48109-0022. Fax: 734936 7026; e-mail: oralh@umich.edu

Received October 1, 2002; revised November 1, 2002; accepted November 7, 2002. cardia $^{7}$ were recorded in the left superior, right superior, and left inferior pulmonary veins.

The left superior and inferior pulmonary veins and the right superior pulmonary vein were electrically disconnected from the left atrium by segmental ostial ablation, guided by recordings of pulmonary vein potentials recorded with the ring catheter. Radiofrequency energy was applied at a maximum power of $35 \mathrm{~W}$, at a target temperature of $52^{\circ} \mathrm{C}$ for $20-45$ seconds, as described previously. ${ }^{8,9}$ Eleven applications of radiofrequency energy were delivered at the ostium of the left superior pulmonary vein. The patient was discharged from the hospital the day after the procedure and was not treated with any antiarrhythmic medications.

During the first month of follow-up, the patient was symptom-free. Subsequently, palpitations recurred and an event monitor revealed that the symptoms were associated with paroxysmal atrial flutter.

An electrophysiology procedure was performed 3 months after the first ablation procedure. After obtaining informed consent, a quadripolar electrode catheter (EP Technologies) was inserted into the right femoral vein and positioned within the coronary sinus. A 20-pole electrode catheter (Daig Minnetonka, MN, USA) was positioned on the lateral right atrial wall. A quadripolar electrode catheter with 2-5-2-mm interelectrode spacing and a 4-mm distal electrode with an embedded thermistor was used for mapping and delivery of radiofrequency energy. The patient presented to the electrophysiology laboratory in sinus rhythm, and atrial flutter that had a cycle length of $220 \mathrm{~ms}$ was reproducibly induced by atrial pacing at a cycle length of $200 \mathrm{~ms}$. Point-by-point endocardial activation and entrainment mapping demonstrated that the atrial flutter originated in the left atrium. The mapping catheter was advanced into the left atrium through the patent foramen ovale. Fractionated electrograms were recorded in close proximity to the left superior pulmonary vein ostium. Pacing at a cycle length of $200 \mathrm{~ms}$ at a site just outside the left superior pulmonary vein resulted 


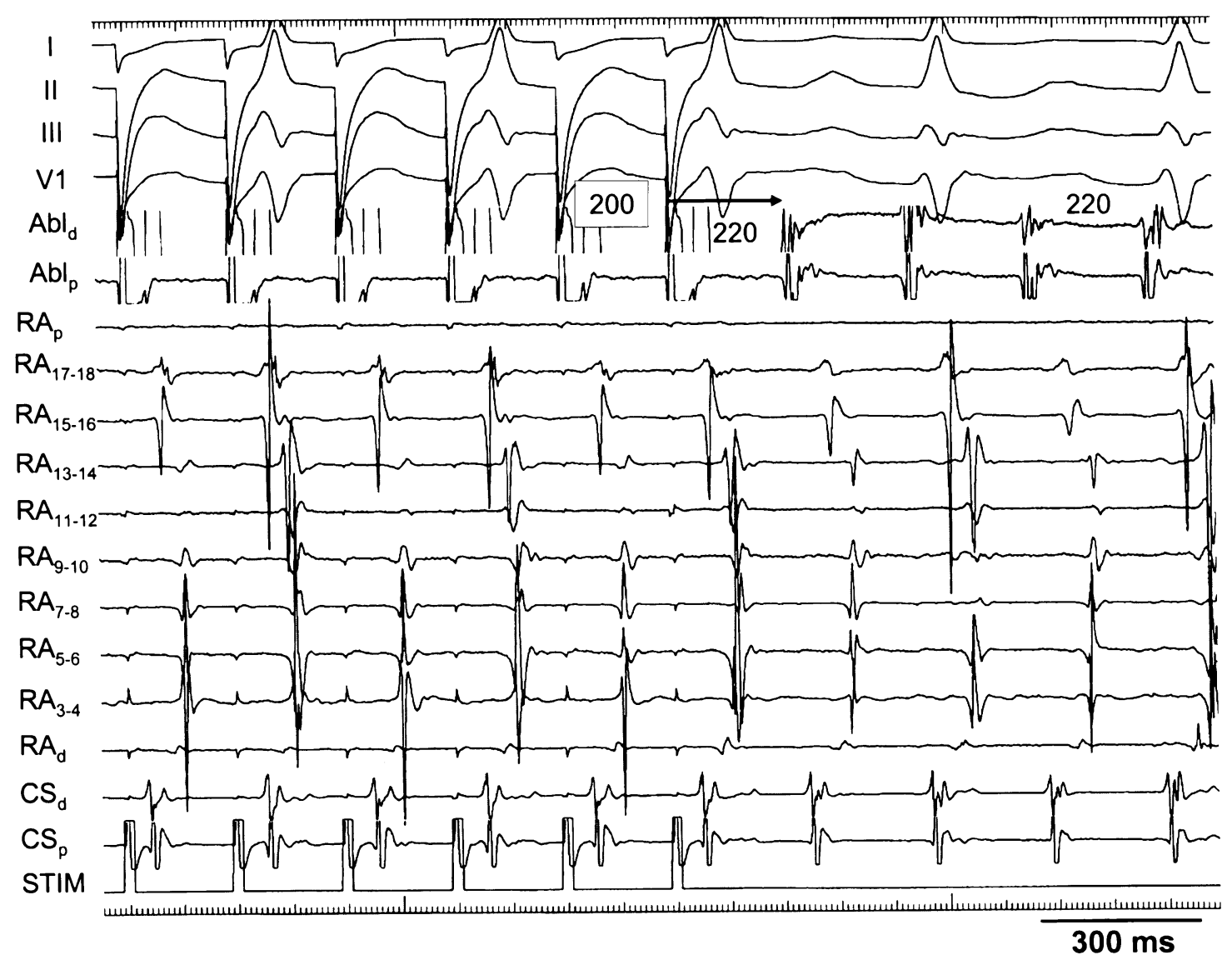

Figure 1. Left atrial flutter, cycle length $220 \mathrm{~ms}$. Surface electrograms (I, II, III, $V_{1}$ ), electrograms from a duodecapolar catheter positioned along the lateral right atrium $\left(R a_{p}, R A_{17-18}, \ldots R A_{3-4}, R A_{d}\right)$, electrograms recorded from the proximal and distal electrode pairs of the quadripolar coronary sinus electrode catheter $\left(C S_{p}\right.$, $\left.C S_{d}\right)$, and the proximal and distal electrode pairs of the quadripolar ablation catheter $\left(A B L_{p}, A B L_{d}\right)$ positioned adjacent to the left superior pulmonary vein ostium, are shown. Pacing from the ablation catheter at a cycle length of 200 ms resulted in concealed entrainment. The postpacing interval (arrow) is equal to the tachycardia cycle length.

in concealed entrainment. The atrial activation sequence during pacing and during tachycardia were identical, and the postpacing interval was equal to the tachycardia cycle length (Fig. 1). A single application of radiofrequency energy at this site resulted in termination of the flutter within 10 seconds (Fig. 2). After ablation, atrial flutter was not inducible either before or during infusion of isoproterenol.

During 3 months follow-up after the second ablation procedure, the patient has had no further symptomatic arrhythmias.

\section{Discussion}

Atrial flutter sometimes originates near the left or right pulmonary vein ostia, particularly in patients with structural heart disease or prior mitral valve surgery. ${ }^{4,10}$ The present case demonstrates that left atrial flutter may be a complication of segmental ostial ablation to isolate a pulmonary vein.
The findings of this case suggest that segmental ostial ablation of the left superior pulmonary vein created a critical isthmus that resulted in left atrial flutter. Because this patient had never had atrial flutter before the ablation procedure, it is likely that the flutter was a postablation manifestation of proarrhythmia.

During segmental ostial ablation, discrete applications of radiofrequency energy generally are necessary at $40 \%-60 \%$ of the circumference of the pulmonary vein ostium. ${ }^{11}$ In the patient described in this report, it is possible that some of the applications of radiofrequency energy were on the venous side of the venoatrial junction, and that others were on the atrial side. These applications of radiofrequency energy may have resulted in a corridor of conducting tissue surrounded by a scar. Successful ablation of the flutter with a single application of radiofrequency energy indicates that the critical isthmus was narrow. 




$300 \mathrm{~ms}$

Figure 2. Termination of atrial flutter by a single application of radiofrequency energy. Radiofrequency energy was delivered at the pacing site shown in Figure 1. The atrial flutter terminated within seconds after the onset of energy delivery, and was no longer inducible after ablation. Catheter positions and abbreviations are as in Figure 1.

Because conventional mapping techniques were used instead of electroanatomic or noncontact mapping, a three-dimensional depiction of the flutter circuit or the critical isthmus is not available. However, the demonstration of concealed entrainment and the response to a single application of radiofrequency energy, provide strong evidence that the critical isthmus was lo-

\section{References}

1. Duru F, Hindricks G, Kottkamp H. Atypical left atrial flutter after intraoperative radiofrequency ablation of chronic atrial fibrillation: Successful ablation using three-dimensional electroanatomic mapping. J Cardiovasc Electrophysiol 2001; 12:602-605.

2. Usui A, Inden Y, Mizutani S, et al. Repetitive atrial flutter as a complication of the left-sided simple maze procedure. Ann Thorac Surg 2002; 73:1457-1459.

3. Mohr FW, Fabricius AM, Falk V, et al. Curative treatment of atrial fibrillation with intraoperative radiofrequency ablation: Short-term and midterm results. J Thorac Cardiovasc Surg 2002; 123:919927.

4. Ouyang F, Ernst S, Vogtmann T, et al. Characterization of reentrant circuits in left atrial macroreentrant tachycardia: Critical isthmus block can prevent atrial tachycardia recurrence. Circulation 2002; 105:1934-1942.

5. Avitall B, Helms RW, Koblish JB, et al. The creation of linear contiguous lesions in the atria with an expandable loop catheter. J Am Coll Cardiol 1999; 33:972-984. cated adjacent to the left superior pulmonary vein.

In conclusion, atrial flutter is a possible complication of segmental ostial ablation to isolate the pulmonary veins. This case demonstrates the importance of documenting the cause of symptoms when symptoms recur after pulmonary vein isolation in patients with atrial fibrillation.

6. Kanagaratnam L, Tomassoni G, Schweikert R, et al. Empirical pulmonary vein isolation in patients with chronic atrial fibrillation using a three-dimensional nonfluoroscopic mapping system: Longterm follow-up. PACE 2001; 24:1774-1779.

7. Oral H, Ozaydin M, Tada H, et al. Mechanistic significance of intermittent pulmonary vein tachycardia in patients with atrial fibrillation. J Cardiovasc Electrophysiol 2002; 13:645-650.

8. Haissaguerre M, Shah DC, Jais P, et al. Electrophysiological breakthroughs from the left atrium to the pulmonary veins. Circulation 2000; 102:2463-2465.

9. Oral H, Knight BP, Tada H, et al. Pulmonary vein isolation for paroxysmal and persistent atrial fibrillation. Circulation 2002; 105:10771081.

10. Jais P, Shah DC, Haissaguerre M, et al. Mapping and ablation of left atrial flutters. Circulation 2000; 101:2928-2934.

11. Oral H, Knight BP, Ozaydin M, et al. Segmental ostial ablation to isolate the pulmonary veins during atrial fibrillation: Feasibility and mechanistic insights. Circulation 2002; 106:1256-1262. 